\title{
Deterministic Chaos: Proposal of an Informal Educational Activity Aimed at High School Students
}

\author{
Valeria Greco ${ }^{*}$, Salvatore Spagnolo \\ Associazione PALERMOSCIENZA c/o Consorzio ARCA viale delle Scienze SN, Italy
}

Copyright (C) 2016 by authors, all rights reserved. Authors agree that this article remains permanently open access under the terms of the Creative Commons Attribution License 4.0 International License

\begin{abstract}
Chaos theory is not present in the Italian school curricula and textbooks in spite of being present in many topics of classical physics and in everyday life. Chaotic dynamics, in fact, are involved in phenomena easily accessible to everyone or in events experienced by most people in their lives (the dripping of a faucet which keeps people awoken at night, meteorology, traffic, population growth). But chaotic phenomena and how they work are ignored. In this paper a series of experiences related to chaos theory are proposed. These experiences can be developed both in an informal and in a formal setting, so that they can be implemented quite simply in schools or in science centres.
\end{abstract}

Keywords Chaos, Informal Education, Double-pendulum, Sinai Billiard

\section{Introduction}

The most important feature of chaos theory is that developed descriptive models have a strong dependence on initial conditions, just as phenomena they aim to describe $[1,2]$. The mathematical models in this field are complicated and typically based on tools or on a cultural background seldom possessed by people. Nonetheless, it is possible to find physical systems whose chaotic evolution is easily viewable through computer simulations or specifically designed experience. In particular, a good starting point to introduce the theory is beginning from everyday experiences.

Of course, a significant learning is characterized by the fact that new concepts or ideas to learn are connected and put into relation with those already possessed by each person. So, the new ideas assume a meaning for everyone because people can increase and sometimes restructure their previous knowledge with new concepts.

On the other hand, transmission of scientific knowledge must use new ways to communicate closer to the citizens and especially young people. Also, it is necessary to recognize the need to create a cultural environment suitable to development of science and characterized by the fall of barriers between science and society.

Moreover, we believe interaction and confrontation are indeed elements leading - if constantly and correctly attended for - to individual and collective growth of people in general and students in particular. So, we start from experiments and go on interacting with students in different ways, analysing an everyday fact or phenomenon to come to understand abstraction. Through this process a significant learning is promoted.

Our experience in informal education develops and grows working with the association PALERMOSCIENZA that promotes growth of scientific communication among young people and citizens outside usual formal structures. In particular, the Association works with students starting from the idea that informal educational activities aim to the development of concepts and to key processes rather than to contents.

Roughly speaking, formal learning is official, curriculum-fixed, scheduled, pedagogically designed with organized learning paths, controlled and often evaluated. Informal learning is unofficial, serving a concrete purpose, unstructured, often uncontrolled and lacking an explicit evaluation.

People learn science not only when, at the end of a cognitive process, master a theory, solve an equation or are able to do practical work in laboratory (typical results of formal learning). Learning science means also being able to build and manage interpretative schemas where to place and interpret scientific phenomena observed every day.

So it is not only important to know the concepts, but also build, use and analyse models, understand a phenomenon and transform it among different representations. This process actualizes itself when people communicate, argue, ask questions, etc. and acquire not only knowledge but, above all, competencies.

\section{Objectives}

The challenge we face as members of 
PALERMOSCIENZA is to create an efficient and valid environment where formal and informal learning converge making significant learning achievable (we operate in Palermo, Italy and in our region, Sicily).

In order to achieve this result, we build situations where experiences and tools from formal and informal learning can be settled in new contexts. So, this environment could stimulate both the investigative attitude and fun inherent to scientific activities.

In particular, the main objectives of our proposal are essentially two. First, the development of an informal experience, aimed at high school students through the inquiry method, allow us to test the interpretative approach to phenomena not present in the school curriculum. The second objective of our proposal is to show that key features of deterministic chaos can be conveyed quite effectively through some simple experiments.

\section{Methods}

We borrow strategies from open inquiry based learning education [3] adapted to the informal learning environment where our experiences take place. So, we put people in a position to tackle questions, issues and controversies from real world, with the aim to solve problems or create solutions in collaboration.

Usually, we pose a problem or a case-study or represent an experience and we ask to people, through an engagement, to design a solution. Then, people are involved in building knowledge through active involvement. In contrast to formal teaching techniques and learning processes, inquiry method gives people deep understanding in addition to the acquisition of knowledge and skills.

Moreover, typically, people has preconceptions about the world. In this sense, the cognitive process stimulated by inquiry tends to eliminate misconceptions present before a deep understanding of the phenomenon. So, the main goal of inquiry method is not only to transfer scientific knowledge, facts, definitions, and concepts, but rather to stimulate people to enhance their ability of reasoning. Moreover this method makes people independent learners capable of identifying main questions and finding relevant answers by a gradual acquisition and expansion of a body of scientific knowledge and abilities.

\section{What We Used}

We present here three activities based on Sinai billiard, a double pendulum and a simple convection example.

These systems involve objects or everyday phenomena with which all people have or have had something to do; on the other hand, they allow some simple visualizations of the concept to be conveyed.

\subsection{First Exhibit}

The first item was a Sinai table, because a pool table is an object of common use among young and not-so-young people, having the opportunity to convey scientific concepts through everyday life experiences shared most likely by all the students. This table has a circular element placed in the middle and, under certain conditions, a little change in the initial position of a ball can cause chaotic behaviour and make its path unpredictable.

Our intention was to involve students in the design of this object. So, we asked to the students of Vincenzo Ragusa e Otama Kiyorama High School of Palermo (Italy) to build the pool table. They built a table lighter and smaller than an ordinary one respecting the ratio of a standard Italian pool table (ratio between sides 1:2). The surface of the table was made by rigid solid wood covered by game table cloth and cushions were of vulcanized rubber without cloth. We spread talcum on the cushions so that the balls rebounded five or six times before stopping.

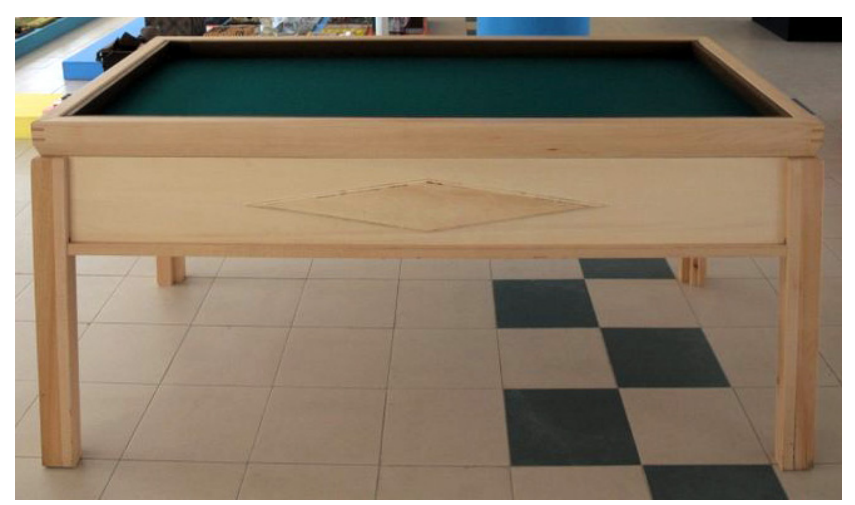

Figure 1. Sinai Table

The students built a wood disc to place in the center of the table to introduce chaotic elements. We knew the table isn't perfect because there is more friction than a real pool table and the Fermat's Principle isn't respected perfectly. However, for us it was more important engaging the students than having a perfect object.

\subsection{Second Exhibit}

A second experience was based on the use of a double pendulum [4] because it allows to identify, within the context of deterministic classical physics, a system that, appropriately modified, manifests chaotic behaviour. Such a system is particularly useful to understand what is the role played by the initial conditions in the dynamics of a physical system and to bust the idea that chaos is present only in many-body systems.

In this case we asked a craftsman to build its arms. $\mathrm{He}$ used polycarbonate plastic for them. We used ball bearings to reduce friction to an absolute minimum between the arms. Also, we chose ordinary material like wood for the support of the pendulum and the table. 


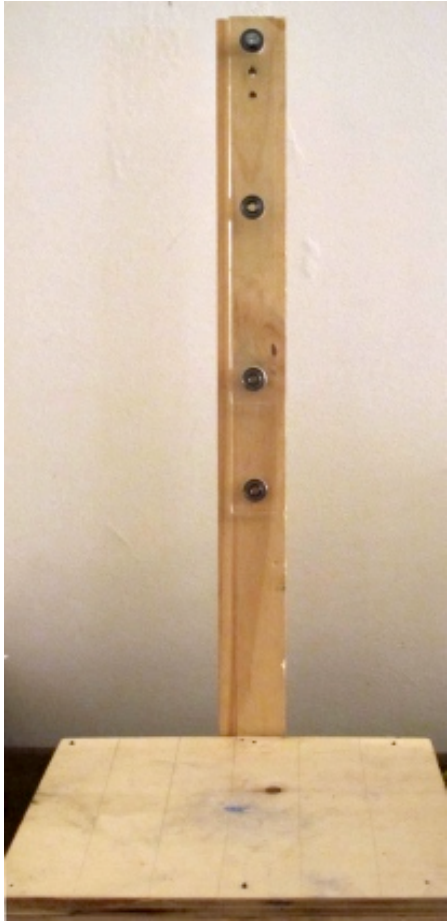

Figure 2. double pendulum

\subsection{Third Exhibit}

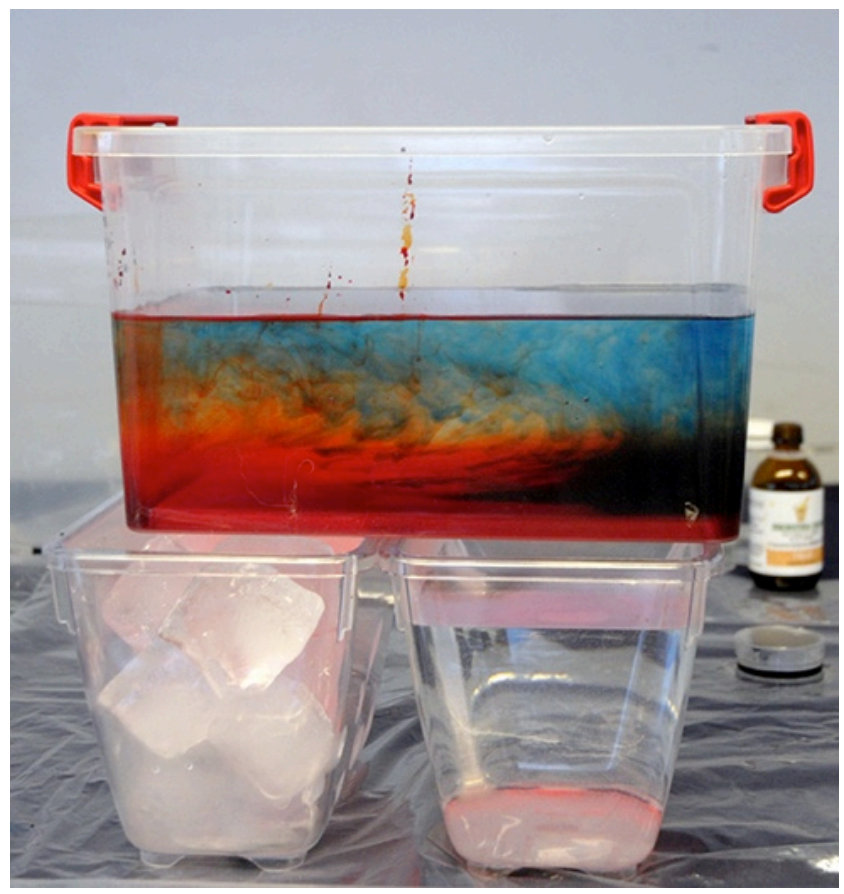

Figure 3. Fluid convection
The last exhibit was an equipment for experiences on fluid convection [5], exploiting pigments diffusion in water. In particular, we used a transparent container filled with water put on two distinct sources of heat. These boundary conditions determine a temperature gradient causing convective motions visible by through colouring pigments placed in water.

Such an experience allows to present the context in which theory of chaos is traditionally introduced looking at thermodynamics and meteorology (E.N. Lorenz, butterfly effect, etc.).

\section{What Has Happened}

We proposed the experiences to a group of fifteen students of the last year of a high school of Palermo (Italy). Starting with the idea that, in informal contexts, theory should emerge from laboratory activities, we used simple questions to present the objects without explaining how they worked to register students' reaction. Here we want to report briefly our activities and to point out the most interesting answers.

We introduced chaos laboratory using some slides and starting from the question "What's chaos?". A student, reported as brilliant by his teacher, gave us an interesting answer: "It is something you cannot explain with a first grade equation". This showed how this student links linearity to a first grade equation. The first example we introduced strengthened this idea because we chose a spring with a mass. In fact, this is a linear system and a little difference in the initial condition produces a small shift in the final position reached in absence of perturbation. The second example was about meteorology, in which systems are notoriously dependent on many variables. For students this is clearly a chaotic system and they gave us as an example many bouncing balls on the floor. We explained it is perfectly possible to have a system with many interacting objects which does not show a chaotic behaviour (for example, a rigid body represented as the continuous version of a large number of atoms strictly connected among them).

Starting from the idea that multi-variables systems could manifest chaos, we chose to introduce the double pendulum.

After a brief presentation about it, we placed the single pieces (the arms, the bolts and the nuts, etc...) of the double pendulum on a table and invited students to assemble them. After some attempts, they realized that in order to have an evident chaotic behaviour, the best solution was to fix the long arm using the first hole. They tried to predict the motion according to what they observed but, after some oscillations, they noted it was impossible. 
Another possible configuration (triple pendulum) was built by the students using the other arm to their disposition. They observed that fixing the long arm in the middle and the other two arms at its two extremes gave back a symmetric and stable situation.

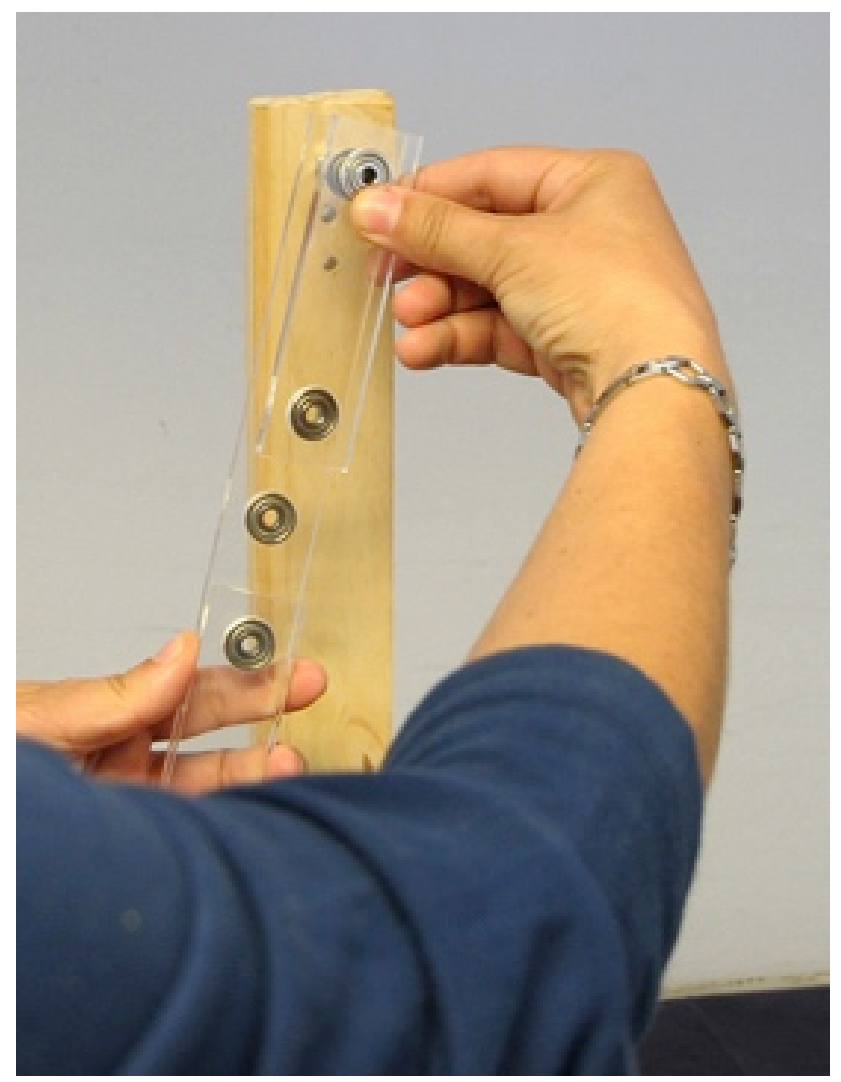

Figure 4. The triple pendulum and its arms

We teased them with the question "Is there a connection between order and chaos?" and, as we expected, all students linked order with predictable events.

In order to bust this idea, we stimulated the students to use the equipment for convection. At first, they kept thinking that at the base of chaotic motion there is mess, but after a thorough observation of the pigment motion in the water, they learnt chaotic motion wouldn't exist without an organization of the water molecules.

We concluded our activity with the Sinai table and a computer simulation to understand why it is impossible to predict the evolution of a chaotic motion.

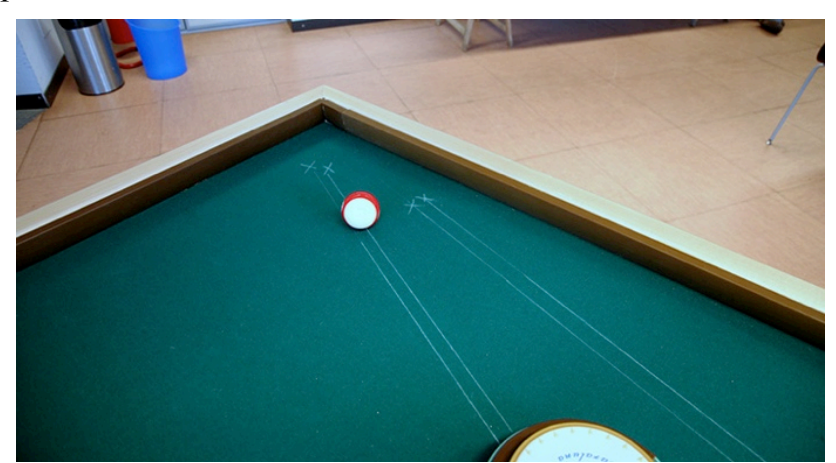

Figure 5. Trajectories on Sinai Table
We drew two paths on the table and we invited the students to play first without the disc in the center and then with it.

In the former case they did many unsuccessful attempts to follow the paths. In fact, they observed in this case, if ball hits disc first, a very small change in the initial conditions "snowballed" and after just two rebounds ball was clearly off the path. If ball hit the table sides without hitting disc, they didn't observe the effect (remember balls can rebound only five or six times on our table).

In order to show a clearer evidence, we used a simple computer application simulating the Sinai table calculating long time trajectories.

\section{Results}

To test efficacy of our approach and understand if our goals had been achieved, we had a brainstorm with students at the end of the experience, asking them several questions: "What did it happen?", "What have you got?", "Did you get the expected results?", "Do you think there is a connection with the life you live every day? Where?".

Sorting out the answers, we point out that some students had found a possible source of chaos in the motion of Solar System planets when you consider interaction among all planets (and not only with the Sun). Other students recognized an analogy between some images present in a slide we showed them during our presentation and a Lorenz attractor they saw during a visit to a science centre. Other students linked chaos to the problem of car traffic in the morning when they go to school, paying attention to dependence from initial time conditions.

The reaction of students supported us on the efficacy of the path we have chosen because they linked the chaos theory with the concepts of non-linearity and linearity in physical systems and found other examples in everyday life.

Also, through this process, the students were involved in the resolution of problems rising from the building of items making them responsible for their learning. We think that this is resonant with the possibility to promote their competences and their significant learning.

\section{Conclusions}

In conclusion, in this paper we have shown three physical experiences whose dynamics, under certain conditions, are nonlinear. The experiments presented here are suitable to be used in a context of informal learning or, suitably modified, in a context of formal learning.

In our opinion, the basic physical concepts communicated through these experiences with students have been observation, statistical uncertainties description and schematisation (models, prediction, interpretation), laws and theories; instead, the basic mathematical concepts conveyed have been relations, functions (representations) and data 
(analysis and forecasting).

Moreover, a topic like chaos has the advantage to have many connections with biology, chemistry and philosophy and to promote the development of many interdisciplinary activities.

The successful experience with the students stimulated us to organize a well-structured laboratory on physical systems manifesting non-linear and linear behaviour depending on initial conditions.

Finally, we want to include these items in a longer lab experience the starting points of which are linear systems, as for example oscillations of simple pendulum or coupled pendulums or fluid dynamics under laminar regime.

\section{Acknowledgements}

We are very grateful to prof. Claudio Fazio of Università degli Studi di Palermo (Italy) for many discussions and suggestions on the subject of this paper, and the association PALERMOSCIENZA for its continuous support.

\section{REFERENCES}

[1] J. Gleick, Chaos: making new science, Viking Books, 1987

[2] J. P. Crutchfield et al, Chaos, Scientific American Vol. 254, 46-57, December1986

[3] Work Package 3 Report: Guide for developing ESTABLISH, Teaching and Learning Units

[4] T. Shinbrot et al, Chaos in a double pendulum, American Journal of Physics Vol. No 60, 491-499, June1992

[5] The mixing offluids, J. Ottimo, Scientific American, Vol. 260, 56-67, Mars 1989 